\title{
Modelling Global Pattern Formations for Collaborative Learning Environments
}

\author{
Corrado Grappiolo, Yun-Gyung Cheong, Rilla Khaled, Georgios N. Yannakakis \\ Centre for Computer Games Research \\ IT University of Copenhagen \\ Rued Langgaards Vej 7 Copenhagen, Denmark \\ Email: \{cogr, yugc, rikh, yannakakis\}@itu.dk
}

\begin{abstract}
We present our research towards the design of a computational framework capable of modelling the formation and evolution of global patterns (i.e. group structures) in a population of social individuals. The framework is intended to be used in collaborative environments, e.g. social serious games and computer simulations of artificial societies. The theoretical basis of our research, together with current state of the art and future work, are briefly introduced.

Index Terms-Global Pattern Formation, Collaborative Learning Environments, Complex Adaptive Systems, Complexity.
\end{abstract}

\section{INTRODUCTION}

The interactions among a population of social individuals (agents) - due to factors such as the agents' attitude, personality, cultural biases and stereotypes, and history of past interactions [1] — yield complex dynamics which lead to the formation of global patterns [2]. Global patterns, which may also be considered as the behaviour of a system as a whole, include group identities and norms, and influence the behaviour of the system's individuals in an indirect, implicit manner [2]. They can generate and aggravate social conflicts, such as discrimination and other forms of inequality. The computational model of global patterns and their impact on the society can serve as an instrument for understanding the causes of social conflicts and for evaluating the effectiveness of intervention methods for conflict resolution. A way to effectively detect global pattern formations is through modelling the local-to-global transition (e.g. from agent interactions to group structures). However, doing so is not a trivial task, since the global structure influences the society-system, creating thus a recursive complex system ${ }^{1}$.

The main questions we intend to answer in this research are: (1) how to model the dynamics of the local-to-global transition? (2) how to predict the formation and evolution of global patterns by characterising their internal structures? (3) what is the nature of the global-to-local transition, and how does its indirect nature influence the agents? (4) how to intervene at the global level (e.g. by means of regulations) in order to affect behaviour at the local level? As our research is informed by the Complex Adaptive Systems (CAS) literature [1], [2], our intention is to leverage the concepts, theories and findings of social and behavioural

\footnotetext{
${ }^{1}$ Sometimes it is referred to as emergence of complexity [1]
}

sciences in identifying the emergent properties of complex systems [3]. Our research is structured into four areas of investigation: (1) exploration of existing theories on global pattern formation; these are usually qualitative and focus on aspects of interaction which are abstract and complex (i.e. collaboration and fairness); (2) formalisation of the theories through computational measures and learning algorithms, and contextualisation of them in realistic social scenarios, such as social dilemmas; (3) implementation of the social scenarios in computer simulations of artificial societies [4], [5]; which will facilitate preliminary validation of our models; (4) extension of application of our models and social scenarios to humanbased collaborative (virtual) environments, such as multiplayer games, which will enable final evaluation of the effectiveness of our models.

As such, global pattern modelling represents a new tool for the creation of educational environments (serious games) that convey concepts like conflict, collaboration, and fairness. Further afield than educational environments, global pattern modelling may contribute to research fields such as behavioural and social sciences, evolutionary dynamics, collective behaviour, as well as macroeconomics and political sciences.

\section{State of OuR Research}

We are currently conducting experiments based on artificial societies to establish optimal learning algorithms and metrics for detecting consolidated group identities (i.e. the ability of an individual to identify himself or herself with his or her group [6]) in the population. The agents interact with each other by means of the social ultimatum bargain game [7]. Several initial configurations representing different group identities (which imply social preferences [8]) have been defined and are being maintained, that is, the agents will not adapt their behaviours in response to behavioural dynamics. We now briefly describe the society's interaction protocol and the structure of the Group Modelling framework (GM) which allows us to detect group identities.

\section{A. The Social Ultimatum Bargain Game in Use}

The interaction protocol is hereby briefly introduced. (1) a population $\mathcal{P}$ of $n$ agents are first divided into $m$ partitions $P$; (2) for each partition, an agent $a_{i}$ is randomly selected to become the provider agent of the social ultimatum bargain 
game, all the other agents of the partition become receiver agents; (3) the provider agent bargains with each receiver agent over an equal endowment $e$. For each bargain, $a_{i}$ proposes an offer $0 \leq o_{i, j} \leq e$ to receiver agent $a_{j}$; according to the social relationship between the agents — which are consequence of the agents' group identities - the offers will vary in generosity [8]; (4) $a_{j}$ can either accept the offer or refuse it; (5) the final outcome for $a_{j}$ is either 0 or $o_{i, j}$; the final outcome for $a_{i}$ is the sum of all the $\left(e-o_{i, j}\right)$ accepted bargains.

\section{B. Measuring Levels of Cooperation}

To detect the existence of sub-groups in the population, and to assign a group identity to the individuals, we analyse the different levels of cooperation amongst the agents through their interactions [6]. Our hypothesis is that by observing the level of cooperation [9] of a provider agent with respect to the whole partition $P_{k}$, we can understand which receiver agents he prefers to bargain with. This would imply that he is more altruistic and collaborative [9] with some of the agents in the partition, hence is more likely to share the same group identity.

For the current state of our research we are considering both deterministic and stochastic metrics of provider agent's favourability which return a value indicating whether the agents under consideration belong to the same group or not (in/out-group values [6]). We will refer to the metrics as interaction classifiers, denoted with $\mathcal{I}$.

\section{Learning Collaboration}

To learn cooperation values among the individuals which encompass the history of past interactions [1] we are currently considering the following Constant- $\alpha$ Monte Carlo update rule for non-stationary environments [10]:

$$
\mathcal{C}_{i, j}(t)=\mathcal{C}_{i, j}(t-1)+\alpha\left[\mathcal{I}_{t}-\mathcal{C}_{i, j}(t)\right]
$$

$\mathcal{C}_{i, j}(t)$ is the cooperation value between the provider agent $a_{i}$ and the receiver agent $a_{j}$ up to the $t$-th interaction, $\mathcal{I}_{t}$ is the level-of-cooperation-value computed by the interaction classifier introduced in Section II-B, and $\alpha$ is a constant stepsize parameter. Finally, collaboration values are obtained by considering reciprocal altruism [1], [9] through the following equation:

$$
\mathcal{C}_{i, j}^{\triangle}(t)=\frac{1}{2}\left(\mathcal{C}_{i, j}+\mathcal{C}_{j, i}\right)
$$

\section{Detecting Group Identities}

The collaboration values $\mathcal{C}_{i, j}^{\triangle}$ are then used as a dissimilarity matrix by a clustering algorithm. The number of group identities and the corresponding agents are then determined. We are currently investigating the use of the complete-link clustering algorithm with elbow rule as validity method.

\section{FUTURE WORK}

Our future work will follow four concurrent paths. First, we will investigate optimal methods and parameters for making the framework robust with respect to the size of the society $(n)$, locality $(m)$, and partial observability of the interactions $(m)$. To this end, we are already in the process of defining a group-based metric of fairness. As fairness of offer distribution is an abstract, subjective, and ambiguous term, we designed several ad-hoc metrics of fairness based on key and generic elements of fair offers, such as the offered amount and the need of the receivers. We then tested these against human notions of fairness, by running a crowdsourcing experiment in which participants were asked to compare levels of fairness of dissimilar offer distribution scenarios in a resource management game environment [11]. Preliminary results suggest that some of our fairness metrics are consistent with human notions of fairness and are both accurate and robust across different game scenarios and levels of fairness distribution complexity. Second, we will enrich the level of complexity of the artificial agents, e.g. by representing affect and enabling adaptation; we will also augment the possibilities of social interactions by introducing other social dilemmas, such as the tragedy of the commons [6]. Third, we will investigate methods for modelling the internal group structures, such as leaderships and norms [12], and the inter-group dynamics (e.g. evolution). We will also investigate methods to intervene at global level (e.g. by modifying the interaction protocols) in order to affect, in a feedback loop, the behaviour of the society, in order to achieve envisaged goals, such as the maximisation of collaboration or the reduction of social inequalities. Finally, we will consider game-based environment, and will increase its level of abstraction by concealing money-related notions and instead representing social conflict scenarios [9].

\section{ACKNOWLEDGMENTS}

This work has been supported, in part, by the FP7 ICT project SIREN (project no: 258453).

\section{REFERENCES}

[1] S. J. Lansing, "Complex Adaptive Systems," Annual Review of Anthropology, vol. 32, pp. 183-204, 2003.

[2] R. Lewin, Complexity: Life at the Edge of Chaos, 2000.

[3] R. L. Goldstone, M. E. Roberts, and T. M. Gureckis, "Emergent Processes in Group Behavior," Current Directions in Psychological Science, vol. 17, pp. 10-15, 2008.

[4] J. M. Epstein and R. L. Axtell, Growing Artificial Societies: Social Science from the Bottom Up (Complex Adaptive Systems), 1996.

[5] J. M. Epstein, "Agent-based computational models and generative social science," Complexity, vol. 4, pp. 41-60, 1999.

[6] R. M. Dawes and D. M. Messick, "Social Dilemmas," International Journal of Psychology, no. 35, pp. 111-116, 2000.

[7] J. N. Bearden, "Ultimatum bargaining experiments: State of the art," Available at SSRN httpssrn comabstract, vol. 626183, pp. 1-56, 2001.

[8] F. Marzo, B. J. Grosz, and A. Pfeffer, "Social preferences in Relational Contexts," in Proceedings of Collective Intentionality, 2005.

[9] Y.-G. Cheong, R. Khaled, C. Grappiolo, J. Campos, C. Martinho, G. P. D. Ingram, A. Paiva, and G. N. Yannakakis, "A Computational Approach Towards Conflict Resolution for Serious Games," in Proceedings of FDG, 2010.

[10] R. S. Sutton and A. G. Barto, Reinforcement Learning: An Introduction (Adaptive Computation and Machine Learning), 1998.

[11] C. Grappiolo, Y. G. Cheong, J. Togelius, R. Khaled, and G. N. Yannakakis, "Towards Player Adaptivity in a Serious Game for Conflict Resolution," in in Proceedings of VS Games, 2011, pp. 192-198.

[12] M. E. J. Newman, "The structure and function of complex networks," vol. 45, pp. 167-256, 2003. 